\title{
The Role of Inflammatory Diet and Vitamin D on the Link between Periodontitis and Cognitive Function: A Mediation Analysis in Older Adults
}

\author{
João Botelho ${ }^{1,2, *}$, Yago Leira ${ }^{3,4,5,6,+}$, João Viana ${ }^{2,+}$, Vanessa Machado ${ }^{1,2}{ }^{\oplus}$, Patrícia Lyra ${ }^{2}$, José Manuel Aldrey ${ }^{7}$, \\ Juan Manuel Pías-Peleteiro ${ }^{7}$, Juan Blanco ${ }^{4,5, \mp}$, Tomás Sobrino ${ }^{6, \ddagger(1)}$ and José João Mendes ${ }^{2, \ddagger(i)}$
}

check for updates

Citation: Botelho, J.; Leira, Y.; Viana, J.; Machado, V.; Lyra, P.; Aldrey, J.M.; Pías-Peleteiro, J.M.; Blanco, J.; Sobrino, T.; Mendes, J.J. The Role of Inflammatory Diet and Vitamin D on the Link between Periodontitis and Cognitive Function: A Mediation Analysis in Older Adults. Nutrients 2021, 13, 924. https://doi.org/ 10.3390/nu13030924

Academic Editor: Přemysl Mladěnka, Remião Fernando and

Mercolini Laura

Received: 18 February 2021

Accepted: 11 March 2021

Published: 12 March 2021

Publisher's Note: MDPI stays neutral with regard to jurisdictional claims in published maps and institutional affiliations.

Copyright: (c) 2021 by the authors. Licensee MDPI, Basel, Switzerland. This article is an open access article distributed under the terms and conditions of the Creative Commons Attribution (CC BY) license (https:/ / creativecommons.org/licenses/by/ $4.0 /)$.
1 Periodontology Department, Instituto Universitário Egas Moniz, 2829-511 Almada, Portugal; vmachado@egasmoniz.edu.pt

2 Evidence-Based Hub, Clinical Research Unit, Centro de Investigação Interdisciplinar Egas Moniz, 2829-511 Almada, Portugal; jpm.viana.1@gmail.com (J.V.); patricialyra10@gmail.com (P.L.); jmendes@egasmoniz.edu.pt (J.J.M.)

3 Periodontology Unit, UCL Eastman Dental Institute and NIHR UCLH Biomedical Research Centre, University College London, London WC1E 6DE, UK; y.leira@ucl.ac.uk

4 Periodontology Unit, Faculty of Medicine and Odontology, University of Santiago de Compostela, 15706 Santiago de Compostela, Spain; jblanco@blancoramos.net

5 Medical-Surgical Dentistry (OMEQUI) Research Group, Health Research Institute of Santiago de Compostela (IDIS), 15706 Santiago de Compostela, Spain

6 Clinical Neurosciences Research Laboratory, Health Research Institute of Santiago de Compostela (IDIS), 15706 Santiago de Compostela, Spain; tomas.sobrino.moreiras@sergas.es

7 Dementia Unit, Department of Neurology, Clinical University Hospital, 15706 Santiago de Compostela, Spain; jaldreyv@yahoo.com (J.M.A.); juan.manuel.pias.peleteiro@gmail.com (J.M.P.-P.)

* Correspondence: jbotelho@egasmoniz.edu.pt; Tel.: +351-212-946-800

+ Equally first authors.

$\ddagger$ Equally last authors.

\begin{abstract}
Patients suffering from periodontitis are at a higher risk of developing cognitive dysfunction. However, the mediation effect of an inflammatory diet and serum vitamin D levels in this link is unclear. In total, 2062 participants aged 60 years or older with complete periodontal diagnosis and cognitive tests from the National Health and Nutrition Examination Survey (NHANES) 20112012 and 2013-2014 were enrolled. The Consortium to Establish a Registry for Alzheimer's disease (CERAD) word learning subtest (WLT) and CERAD delayed recall test (DRT), the animal fluency test (AFT) and the digit symbol substitution test (DSST) was used. Dietary inflammatory index (DII) was computed via nutrition datasets. Mediation analysis tested the effects of DII and vitamin D levels in the association of mean probing depth (PD) and attachment loss (AL) in all four cognitive tests. Periodontitis patients obtained worse cognitive test scores than periodontally healthy individuals. DII was negatively associated with CERAD-WLT, CERAD-DRT, AFT and DSST, and was estimated to mediate between $9.2 \%$ and $36.4 \%$ of the total association between periodontitis with cognitive dysfunction $(p<0.05)$. Vitamin D showed a weak association between CERAD-DRT, AFT and DSST and was estimated to between $8.1 \%$ and $73.2 \%$ of the association between periodontitis and cognitive dysfunction $(p<0.05)$. The association between periodontitis and impaired cognitive function seems to be mediated both by a proinflammatory dietary load and vitamin D deficiency. Future studies should further explore these mediators in the periodontitis-cognitive decline link.
\end{abstract}

Keywords: periodontitis; periodontal disease; inflammation; vitamin D; diet; oral health

\section{Introduction}

Dementia is a syndrome of progressive memory loss and impaired cognitive ability, impacting everyday activities [1]. This condition is estimated to affect 131.5 million people worldwide by 2050, with severe impairment on patients' quality of life [2]. Currently, the 
main focus of dementia research relies on its neurological characteristics, mechanisms and novel therapeutic breakthroughs [3]. To date, understanding dementia and its risk factors has been greatly accomplished through large population-based surveys [3,4], whose results have expanded knowledge on new oral health-related risk factors, such as tooth loss [5-7] and periodontitis [8-13].

Periodontitis is an irreversible disease that can lead to tooth loss $[14,15]$. This noncommunicable disease is characterized by a polymicrobial dysbiotic infection of the periodontium [16,17]. In cases of unresolved periodontitis, teeth may be lost entirely and deteriorate quality of life in a reversible manner if treatment is delivered [18]. Despite the association between periodontitis and dementia being far from fully comprehended [19], demented patients tend to have worse periodontal status $[8,13,20]$, and systemic inflammation may mediate the association between periodontitis and cognitive impairment [8].

Likewise, evidence shows an apparent role of diet in both dementia and periodontitis. Poor diet and vitamin B deficits are associated with cognitive decline [21,22]. In fact, adherence to proinflammatory dietary patterns was associated with greater cognitive decline $[23,24]$, while dietary anti-inflammatory patterns reduce the inflammatory burden in periodontitis lesions [25]. Additionally, vitamin D deficiency has been growing attention reasonably in dementia [26-28] and periodontitis [29] since lower vitamin D intake associates with a higher risk of both conditions. However, the mediating role of inflammatory diet and vitamin $\mathrm{D}$ on the association of periodontal status and cognitive function has never been investigated.

Therefore, we aimed to explore the association of periodontitis with cognitive functioning and the mediation effect of an inflammatory diet and serum vitamin $\mathrm{D}$ levels in this link.

\section{Materials and Methods}

\subsection{Study Design and Participants}

A secondary analysis was carried out on two datasets from the National Health and Nutrition Examination Survey (NHANES). NHANES is a stratified multistage national representative survey with a civilian noninstitutionalized population in fifty states of the USA and the District of Columbia. All details on sampling, design, medical records and periodontal data collections can be found at www.cdc.gov/nchs/nhanes.htm (accessed on 30 August 2020). Both NHANES 2011-2012 and 2013-2014 datasets were reviewed and approved by the Centers for Disease Control (CDC) and Prevention National Increase for Health Statistics Research (NCHS) Ethics Review Board, and all included participants provided written informed consent. In the NHANES 2011-2014, a subsample of the population aged 60 years and above were asked to carry out cognitive tests. The survey also included a periodontal examination in all dentition. For the purpose of this study, we have included those participants that completed both periodontal examination and cognitive function tests.

This study followed the strengthening of the reporting of observational studies in epidemiology (STROBE) guidelines [30] (Supplementary Table S1).

\subsection{Cognitive Assessment}

Cognitive function was measured using a series of assessments [31], including (1) word learning and recall modules from the Consortium to Establish a Registry for Alzheimer's disease (CERAD) [32]; (2) the animal fluency test [33]; and (3) the Digit Symbol Substitution test (DSST) [34].

The CERAD word learning subtest (CERAD-WLT) assesses immediate and delayed learning ability for new verbal information (memory sub-domain) [32]. The test consists of three consecutive learning trials and a delayed recall. For the learning trials, participants are instructed to read aloud 10 unrelated words, one at a time, as they are presented. Immediately following the presentation of the words, participants recall as many words as possible. In each of the three learning trials, the order of the 10 words is changed. The 
maximum score possible on each trial is 10. In NHANES, the words for the learning trials were presented in large, bolded letters on a computer monitor. Participants, who were unable to read due to literacy or a visual impairment, were asked to repeat each word after it was read by the interviewer. The delayed word recall (CERAD delayed recall test (CREAD-DRT)) occurred after the other two cognitive exercises (animal fluency and DSST) were completed (approximately 8-10 min from the start of the word learning trials).

The animal fluency test examines categorical verbal fluency, a component of executive function [33]. Participants are asked to name as many animals as possible in one minute. A point is given for each named animal. In NHANES, participants first were asked to name three items of clothing, another verbal fluency category, as a practice test. Participants who could not name three articles of clothing did not continue with the animal fluency exercise.

The digit symbol substitution test (DSST) relies on processing speed, sustained attention, and working memory [34]. The exercise is conducted using a paper form that has a key at the top containing 9 numbers paired with symbols. Participants have 2 min to copy the corresponding symbols in the 133 boxes that adjoin the numbers. The score is the total number of correct matches. A sample practice test is administered before the participants begin the main test. In NHANES, participants who could not correctly match the symbols with the numbers on their own during the pretest practice did not continue.

\subsection{Periodontal Assessment}

A full-mouth periodontal examination was conducted by calibrated examiners as described elsewhere [35]. Pocket depth (PD) and attachment loss (AL) were recorded at six sites per tooth (mesio-buccal, mid-buccal, disto-buccal, mesio-lingual (or palatal), mid-lingual (or palatal), and disto-lingual (or palatal)). Periodontitis was defined as the presence of a minimum of 2 or more sites with $\mathrm{AL} \geq 3 \mathrm{~mm}$ and PD $\geq 4 \mathrm{~mm}$ or one site with $P D \geq 5 \mathrm{~mm}$ [36]. The number of missing teeth was also recorded.

\subsection{Dietary Inflammatory Index (DII)}

The DII is a literature-based instrument [37] that computes the inflammatory properties of a diet, based on the association of certain food and dietary constituents on defined inflammatory hallmarks: CRP, TNF- $\alpha$ and IL-1 $\beta$, IL-4, IL-6 and IL-10. From a possible total of 45 food parameters, the final score is a continuous measure, interpreted as strongly anti-inflammatory (the lowest score) to strongly proinflammatory (the highest score), respectively [37].

In this study, DII was computed using the NHANES database for a dietary interview for the total nutrient intakes. We calculated the DII score for the 26 food parameters available. Eugenol, garlic, ginger, niacin, onion, saffron, saturated fat, trans fat, turmeric, green/black tea, flavan-3-ol, flavones, flavonols, flavanones, anthocyanidins, isoflavones, pepper, thyme/oregano, rosemary were not included because no information was available. First, we calculated the z-score of each food parameter and for each participant. Second, each individual z-score was converted to a centered percentile. Third, each centered percentile was multiplied by the standardized overall inflammatory effect score [37]. Finally, the DII score was summed and obtained for each participant.

\subsection{Sociodemographic and Health-Related Variables}

In the sociodemographic variables, we included age, gender, ethnicity, educational level, marital status and family income to poverty ratio. Current smokers were those who had smoked $\geq 100$ cigarettes during their lifetime and were still active smokers. Former smokers were defined as those who had smoked $\geq 100$ cigarettes during their lifetime but had stopped. Those who had smoked $<100$ cigarettes during their lifetimes were categorized as never smokers. Minutes of sedentarism was used as a proxy of physical activity.

To assess participant's systemic status, we considered the number of self-reported medical conditions as an aggregate continuous variable to note the presence of asthma, 
congestive heart failure, coronary heart disease, angina, stroke, heart attack, emphysema, overweight, bronchitis, liver conditions, thyroid conditions and cancer. Considering the strong association of hypertension [38] and diabetes [39,40] with periodontitis in the NHANES, we included these variables separately. The presence of hypertension was defined according to the presence of average systolic blood pressure $>140 \mathrm{mmHg}$ or average diastolic blood pressure $>90 \mathrm{mmHg}$ [41]. The presence of diabetes was recorded as a self-reported measure from a previous report of a doctor or a health professional. Body mass index (BMI) was calculated using the standard formula weight/height $[2,42]$.

\subsection{Biochemical Parameters}

Data from lipid fractions (including HDL cholesterol (mg/dL), LDL cholesterol $(\mathrm{mg} / \mathrm{dL})$, total cholesterol $(\mathrm{mg} / \mathrm{dL})$, and triglycerides $(\mathrm{mg} / \mathrm{dL}))$ were analyzed from blood specimens $[43,44]$. WBC count $\left(10^{9} / \mathrm{L}\right)$ were analyses through volume, conductivity and scatter technology [43,44]. In addition, serum levels of total vitamin D (nmol/L) [43,44] were calculated by the sum of 25-hydroxyvitamin D2 and 25-hydroxyvitamin D3.

\subsection{Statistical Analysis}

Datasets from the NHANES 2011-2012 and 2013-2014 were handled through SPSS version 25.0 for Macintosh (Armonk, NY, USA, IBM Corp.). Concerning the periodontal diagnosis, data were exported to a specific MO Excel with appropriate algorithms that were derived from formulating the periodontal case definition [45,46]. We report continuous and categorical variables through mean \pm standard deviation (SD) and a number of cases (n) with percentage (\%), respectively. All statistical analyses were computed in R. After inspection of data normality and homoscedasticity; we employed the t-test to compare mean values according to the periodontal status. Additionally, the chi-squared test was used for the comparison of categorical variables.

Finally, a mediation analysis was carried out to examine the mediating effect of DII and vitamin D levels in the association of two periodontal measures (mean PD and Mean $\mathrm{AL}$ ) with each cognitive functioning test (CERAD-WLT, CERAD-DRT, animal fluency and DSST) (Figure 1). The mediating effect of DII and vitamin D in the association of PD and CAL with all four cognitive function tests were carried out using the r package "lavaan". Mediation analysis was done through the establishment of three pathways (a, b and c) (Figure 1). For this purpose, we set three pathways: (1) exposure to mediator; (2) mediator to outcome (direct effect); and (3) exposure to outcome (total effect). The total effect reflected the sum of a direct effect and a mediated (indirect) effect. The percentage of the mediated effect was calculated using the following formula: (mediated effect/total effect) $\times 100$ [47]. Bootstrapping was used for significance testing for mediation analysis.

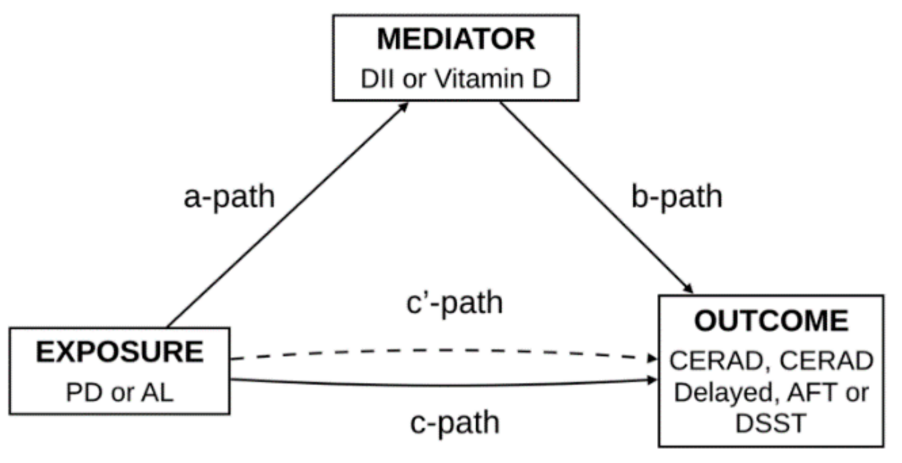

Figure 1. Path diagram of the mediation analysis models.

A significance level of 5\% was set in all inferential analyses. 


\section{Results}

\subsection{General Characteristics}

From 3054 participants that have completed cognitive function tests, 2062 had complete periodontal data and were included in our analyses (Figure 2).

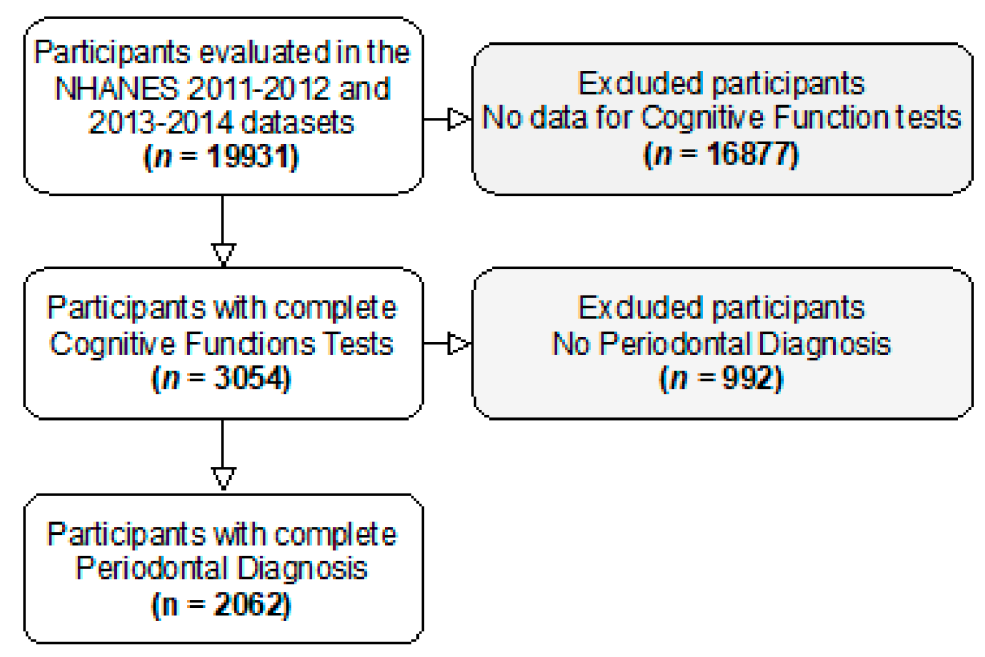

Figure 2. Participants flowchart.

Table 1 presents general characteristics of study participants weighted to the US population according to the presence/absence of periodontitis.

Statistically significant differences were observed between those with and without periodontitis in terms of sociodemographic data (i.e., gender, race, education level, marital status and family income/poverty ratio) (all $p<0.001$ ).

Compared to non-periodontitis individuals, participants diagnosed with periodontitis were more frequently current smokers $(14.4 \%$ vs. $5.0 \%, p<0.001)$, had more often a previous history of hypertension ( $30.9 \%$ vs. $25.5 \%, p=0.014)$ and diabetes $(26.7 \%$ vs. $15.8 \%$, $p=0.001$ ), and were less physically active (minutes of sedentarism: 462.2 vs. $435.4 \mathrm{~min}$, $p<0.001$ ).

As expected, clinical parameters of periodontal inflammation and destruction were increased in the periodontal group (PD: 1.87 vs. $1.13 \mathrm{~mm}, p<0.001$ and AL: 2.51 vs. $1.34 \mathrm{~mm}, p<0.001)$.

Cognitive performance of periodontitis subjects was significantly worse than periodontally healthy participants (CERAD-WLT: 17.2 vs. 19.2, $p<0.001$; CERAD-DRT: 5.4 vs. $6.2, p<0.001$; animal fluency test: 15.0 vs. $17.1, p<0.001$; DSST: 40.9 vs. $50.2, p<0.001$ ).

A more proinflammatory diet was observed in periodontitis subjects (DII: $-0.05 \mathrm{vs}$. $-0.32, p<0.001)$. Likewise, the biochemical analysis showed that peripheral levels of vitamin D and HDL were decreased, while WBC counts were elevated in the periodontitis group compared to those without periodontitis (all $p<0.001$ ).

\subsection{Mediation Analysis}

\subsubsection{Proinflammatory Diet}

There was evidence that the association between periodontitis and poor performance in the four cognitive tests was mediated by a proinflammatory diet.

Mean PD and AL were negatively associated with animal fluency test and DSST and positively associated with DII (Tables $2-5$ ). Further, DII was negatively associated with CERAD-WLT, CERAD-DRT, animal fluency test and DSST (Tables 2-5). It was estimated that between $9.2 \%$ and $36.4 \%$ of the total association between periodontitis with cognitive dysfunction was mediated by DII $(p<0.05)$. 
Table 1. Sample characteristics according to periodontal status $(n=2062)$.

\begin{tabular}{|c|c|c|c|}
\hline & $\begin{array}{l}\text { No Periodontitis } \\
\quad(n=625)\end{array}$ & $\begin{array}{l}\text { Periodontitis } \\
\quad(n=1437)\end{array}$ & $p$-Value \\
\hline Age (years), mean (SD) & $68.74(0.27)$ & $69.0(0.18)$ & 0.395 \\
\hline \multicolumn{4}{|l|}{ Gender, $n(\%)$} \\
\hline Males & $228(36.5)$ & $816(56.8)$ & \multirow[b]{2}{*}{$<0.001$} \\
\hline Females & $397(63.5)$ & $621(43.2)$ & \\
\hline \multicolumn{4}{|l|}{ Race/ethnicity, $n(\%)$} \\
\hline Mexican American & $50(8.0)$ & $162(11.2)$ & \\
\hline Non-Hispanic White & $46(7.3)$ & $173(12.0)$ & \\
\hline Non-Hispanic Black & $373(59.7)$ & $559(38.9)$ & $<0.001$ \\
\hline Other Hispanic & $92(14.7)$ & $371(25.8)$ & \\
\hline Other race & $64(10.2)$ & $172(12)$ & \\
\hline \multicolumn{4}{|l|}{ Education level, $n(\%)$} \\
\hline$<$ High school & $39(6.2)$ & 215 (14.9) & \\
\hline High school & $179(28.6)$ & $523(36.3)$ & $<0.001$ \\
\hline >High school & $407(65.1)$ & $699(48.6)$ & \\
\hline \multicolumn{4}{|l|}{ Smoking status, $n(\%)$} \\
\hline Never & $390(62.4)$ & $687(48.5)$ & \\
\hline Former & $204(32.6)$ & $530(36.9)$ & \\
\hline Current & $31(5.0)$ & $209(14.6)$ & \\
\hline BMI $\left(\mathrm{kg} / \mathrm{m}^{2}\right)$, mean $(\mathrm{SD})$ & $28.8(0.24)$ & $28.7(0.16)$ & 0.408 \\
\hline Family income/poverty ratio, mean (SD) & $2.98(1.69)$ & $2.40(1.62)$ & $<0.001$ \\
\hline \multicolumn{4}{|l|}{ Marital status } \\
\hline Single & $39(6.2)$ & $94(6.5)$ & \\
\hline Married/living with a partner & $404(64.6)$ & $831(57.8)$ & 0.012 \\
\hline Divorced/separated/widowed & $182(29.1)$ & $512(35.6)$ & \\
\hline Chronic medical conditions, mean (SD) & $0.73(0.44)$ & $0.75(0.43)$ & 0.351 \\
\hline Diabetes, $n(\%)$ & $99(15.8)$ & $340(26.7)$ & 0.001 \\
\hline Hypertension, $n(\%)$ & $159(25.5)$ & 439 (30.9) & 0.014 \\
\hline Minutes of sedentarism (min), mean (SD) & $435.4(577.4)$ & $462.2(895.7)$ & $<0.001$ \\
\hline \multicolumn{4}{|l|}{ Cognitive function, mean (SD) } \\
\hline CERAD word learning & $19.2(6.1)$ & $17.2(6.8)$ & $<0.001$ \\
\hline CERAD delayed recall & $6.2(2.6)$ & $5.4(2.7)$ & $<0.001$ \\
\hline Animal fluency test & $17.1(6.7)$ & $15.0(6.9)$ & $<0.001$ \\
\hline DSST & $50.2(20.4)$ & $40.9(21.1)$ & $<0.001$ \\
\hline \multicolumn{4}{|l|}{ Periodontal measurements, mean (SD) } \\
\hline $\mathrm{PD}(\mathrm{mm})$ & $1.13(0.32)$ & $1.87(0.80)$ & $<0.001$ \\
\hline $\mathrm{AL}(\mathrm{mm})$ & $1.34(0.42)$ & $2.51(1.23)$ & $<0.001$ \\
\hline Missing teeth & $6.9(7.3)$ & $9.1(7.2)$ & $<0.001$ \\
\hline DII, mean (SD) & $-0.32(1.81)$ & $-0.05(1.80)$ & $<0.001$ \\
\hline \multicolumn{4}{|l|}{ Biochemical parameters, mean (SD) } \\
\hline Vitamin D (nmol/L) & $82.92(30.47)$ & $72.87(30.67)$ & $<0.001$ \\
\hline $\operatorname{WBC}\left(10^{9} / \mathrm{L}\right)$ & $6.57(1.79)$ & $7.02(2.56)$ & $<0.001$ \\
\hline $\mathrm{HDL}(\mathrm{mg} / \mathrm{dL})$ & $57.57(16.29)$ & $55.20(16.22)$ & $<0.001$ \\
\hline $\mathrm{LDL}(\mathrm{mg} / \mathrm{dL})$ & $112.00(37.27)$ & $110.07(36.49)$ & 0.438 \\
\hline Total cholesterol (mg/dL) & $193.59(42.49)$ & $190.68(41.06)$ & 0.065 \\
\hline Triglycerides (mg/dL) & $118.07(63.25)$ & $121.25(73.92)$ & 0.505 \\
\hline
\end{tabular}

Abbreviations: BMI, body mass index; CERAD, Consortium to Establish a Registry for Alzheimer's disease; DSST, digit symbol substitution test; AL, attachment loss; PD, pocket depth; WBC, white blood cells; DII, dietary inflammatory index; HDL: high-density lipoprotein; LDL: low-density lipoprotein. 
Table 2. Estimates (SE) of mediation analysis for the association between periodontitis and CERAD word learning test.

\begin{tabular}{|c|c|c|c|c|c|c|}
\hline \multicolumn{7}{|c|}{ Exposure: PD and Outcome: CERAD Word Learning Test } \\
\hline Mediator & $\begin{array}{l}\text { Exposure to } \\
\text { Mediator }\end{array}$ & $\begin{array}{l}\text { Mediator to } \\
\text { Outcome }\end{array}$ & Direct Effect & $\begin{array}{c}\text { Mediated } \\
\text { (Indirect) } \\
\text { Effect }\end{array}$ & $\begin{array}{l}\text { Total Effect } \\
\text { (Exposure to } \\
\text { Outcome) }\end{array}$ & $\begin{array}{c}\text { Proportion } \\
\text { Mediated (\%) }\end{array}$ \\
\hline DII & $0.21(0.08) * *$ & $-0.37(0.11)^{* * *}$ & $-0.41(0.25)$ & $-0.08(0.04) *$ & $-0.48(0.25)$ & 16.2 \\
\hline $\begin{array}{l}\text { Vitamin D } \\
(\mathrm{nmol} / \mathrm{L})\end{array}$ & $-6.24(1.29)^{* * *}$ & $0.01(0.01)$ & $-0.36(0.27)$ & $-0.06(0.04)$ & $-0.42(0.27)$ & - \\
\hline \multicolumn{7}{|c|}{ Exposure: AL and Outcome: CERAD Word Learning Test } \\
\hline Mediator & $\begin{array}{l}\text { Exposure to } \\
\text { Mediator }\end{array}$ & $\begin{array}{l}\text { Mediator to } \\
\text { Outcome }\end{array}$ & Direct Effect & $\begin{array}{c}\text { Mediated } \\
\text { (Indirect) } \\
\text { Effect }\end{array}$ & $\begin{array}{c}\text { Total Effect } \\
\text { (Exposure to } \\
\text { Outcome) }\end{array}$ & $\begin{array}{c}\text { Proportion } \\
\text { Mediated (\%) }\end{array}$ \\
\hline DII & $0.14(0.05) * *$ & $-0.37(0.11)^{* * *}$ & $-0.20(0.17)$ & $-0.05(0.02) *$ & $-0.26(0.17)$ & 19.9 \\
\hline $\begin{array}{l}\text { Vitamin D } \\
(\mathrm{nmol} / \mathrm{L})\end{array}$ & $-3.80(0.84)^{* * *}$ & $0.05(0.03)$ & $-0.24(0.18)$ & $-0.04(0.03)$ & $-0.28(0.18)$ & - \\
\hline
\end{tabular}

All models adjusted for sociodemographic variables (age, gender, race, education, marital status, family income/poverty ratio), health behaviors (minutes of sedentarism and smoking habit), body mass index, missing teeth and systemic status (number of chronic medical conditions, hypertension, diabetes, HDL cholesterol, LDL cholesterol, total cholesterol, triglycerides). ${ }^{*} p<0.05 ;{ }^{* *} p<0.01 ;{ }^{* * *} p<0.001$. Abbreviations: AL, attachment loss; PD, pocket depth; DII, dietary inflammatory index; CERAD, Consortium to Establish a Registry for Alzheimer's disease.

Table 3. Estimates (SE) of mediation analysis for the association between periodontitis and CERAD Delayed Recall Test.

\begin{tabular}{|c|c|c|c|c|c|c|}
\hline \multicolumn{7}{|c|}{ Exposure: PD and Outcome: CERAD Delayed Recall Test } \\
\hline Mediator & $\begin{array}{l}\text { Exposure to } \\
\text { Mediator }\end{array}$ & $\begin{array}{l}\text { Mediator to } \\
\text { Outcome }\end{array}$ & Direct Effect & $\begin{array}{c}\text { Mediated } \\
\text { (Indirect) } \\
\text { Effect }\end{array}$ & $\begin{array}{l}\text { Total Effect } \\
\text { (Exposure to } \\
\text { Outcome) }\end{array}$ & $\begin{array}{c}\text { Proportion } \\
\text { Mediated (\%) }\end{array}$ \\
\hline DII & $0.21(0.08) * *$ & $-0.13(0.05)^{* * *}$ & $-0.10(0.11)$ & $-0.03(0.01) *$ & $-0.12(0.11)$ & 22.8 \\
\hline $\begin{array}{l}\text { Vitamin D } \\
(\mathrm{nmol} / \mathrm{L})\end{array}$ & $-6.24(1.29)^{* * *}$ & $0.01(0.00)^{*}$ & $-0.06(0.11)$ & $-0.05(0.02) *$ & $-0.11(0.11)$ & 73.2 \\
\hline \multicolumn{7}{|c|}{ Exposure: AL and Outcome: Delayed Recall Test } \\
\hline Mediator & $\begin{array}{l}\text { Exposure to } \\
\text { Mediator }\end{array}$ & $\begin{array}{l}\text { Mediator to } \\
\text { Outcome }\end{array}$ & Direct Effect & $\begin{array}{c}\text { Mediated } \\
\text { (Indirect) } \\
\text { Effect }\end{array}$ & $\begin{array}{c}\text { Total Effect } \\
\text { (Exposure to } \\
\text { Outcome) }\end{array}$ & $\begin{array}{c}\text { Proportion } \\
\text { Mediated (\%) }\end{array}$ \\
\hline DII & $0.14(0.05)^{* *}$ & $-0.13(0.05)^{* *}$ & $-0.03(0.07)$ & $-0.02(0.01) *$ & $-0.05(0.07)$ & 36.4 \\
\hline $\begin{array}{l}\text { Vitamin D } \\
(\mathrm{nmol} / \mathrm{L})\end{array}$ & $-3.80(0.84)^{* * *}$ & $0.01(0.00)^{*}$ & $-0.04(0.07)$ & $-0.03(0.01)^{*}$ & $-0.06(0.07)$ & 63.3 \\
\hline
\end{tabular}

All models adjusted for sociodemographic variables (age, gender, race, education, marital status, family income/poverty ratio), health behaviors (minutes of sedentarism and smoking habit), body mass index, missing teeth and systemic status (number of chronic medical conditions, hypertension, diabetes, HDL cholesterol, LDL cholesterol, total cholesterol, triglycerides). ${ }^{*} p<0.05 ;{ }^{* *} p<0.01 ;{ }^{* * *} p<0.001$. Abbreviations: AL, attachment loss; PD, pocket depth; DII, dietary inflammatory index; CERAD, Consortium to Establish a Registry for Alzheimer's disease. 
Table 4. Estimates (SE) of mediation analysis for the association between periodontitis and animal fluency test.

\begin{tabular}{|c|c|c|c|c|c|c|}
\hline \multicolumn{7}{|c|}{ Exposure: PD and Outcome: Animal Fluency Test } \\
\hline Mediator & $\begin{array}{l}\text { Exposure to } \\
\text { Mediator }\end{array}$ & $\begin{array}{l}\text { Mediator to } \\
\text { Outcome }\end{array}$ & Direct Effect & $\begin{array}{c}\text { Mediated } \\
\text { (Indirect) } \\
\text { Effect }\end{array}$ & $\begin{array}{l}\text { Total Effect } \\
\text { (Exposure to } \\
\text { Outcome) }\end{array}$ & $\begin{array}{c}\text { Proportion } \\
\text { Mediated (\%) }\end{array}$ \\
\hline DII & $0.21(0.08)^{* *}$ & $-0.46(0.11)^{* * *}$ & $-0.49(0.27)$ & $-0.10(0.05) *$ & $-0.58(0.27) *$ & 16.7 \\
\hline $\begin{array}{l}\text { Vitamin D } \\
(\mathrm{nmol} / \mathrm{L})\end{array}$ & $-6.24(1.29)^{* * *}$ & $0.01(0.01) *$ & $-0.42(0.28)$ & $-0.08(0.05) *$ & $-0.51(0.28)$ & 12.2 \\
\hline \multicolumn{7}{|c|}{ Exposure: AL and Outcome: Animal Fluency Test } \\
\hline Mediator & $\begin{array}{l}\text { Exposure to } \\
\text { Mediator }\end{array}$ & $\begin{array}{l}\text { Mediator to } \\
\text { Outcome }\end{array}$ & Direct Effect & $\begin{array}{c}\text { Mediated } \\
\text { (Indirect) } \\
\text { Effect }\end{array}$ & $\begin{array}{l}\text { Total Effect } \\
\text { (Exposure to } \\
\text { Outcome) }\end{array}$ & $\begin{array}{c}\text { Proportion } \\
\text { Mediated (\%) }\end{array}$ \\
\hline DII & $0.14(0.05) * *$ & $-0.46(0.11)^{* * *}$ & $-0.32(0.18)$ & $-0.07(0.03) *$ & $-0.39(0.18) *$ & 16.5 \\
\hline $\begin{array}{l}\text { Vitamin D } \\
(\mathrm{nmol} / \mathrm{L})\end{array}$ & $-3.80(0.84)^{* * *}$ & $0.01(0.01)$ & $-0.34(0.18)$ & $-0.05(0.03)$ & $-0.39(0.18) *$ & - \\
\hline
\end{tabular}

All models adjusted for sociodemographic variables (age, gender, race, education, marital status, family income/poverty ratio), health behaviors (minutes of sedentarism and smoking habit), body mass index, missing teeth and systemic status (number of chronic medical conditions, hypertension, diabetes, HDL cholesterol, LDL cholesterol, total cholesterol, triglycerides). ${ }^{*} p<0.05 ;{ }^{* *} p<0.01 ;{ }^{* * *} p<0.001$. Abbreviations: AL, attachment loss; PD, pocket depth; DII, dietary inflammatory index.

Table 5. Estimates (SE) of mediation analysis for the association between periodontitis and DSST.

\begin{tabular}{|c|c|c|c|c|c|c|}
\hline \multicolumn{7}{|c|}{ Exposure: PD and Outcome: DSST } \\
\hline Mediator & $\begin{array}{l}\text { Exposure to } \\
\text { Mediator }\end{array}$ & $\begin{array}{l}\text { Mediator to } \\
\text { Outcome }\end{array}$ & Direct Effect & $\begin{array}{c}\text { Mediated } \\
\text { (Indirect) } \\
\text { Effect }\end{array}$ & $\begin{array}{l}\text { Total Effect } \\
\text { (Exposure to } \\
\text { Outcome) }\end{array}$ & $\begin{array}{c}\text { Proportion } \\
\text { Mediated (\%) }\end{array}$ \\
\hline DII & $0.21(0.08)^{* *}$ & $-1.70(0.30)^{* * *}$ & $-3.23(0.72)^{* * *}$ & $-0.36(0.16) *$ & $-3.24(0.72) * * *$ & 11.0 \\
\hline $\begin{array}{l}\text { Vitamin D } \\
(\mathrm{nmol} / \mathrm{L})\end{array}$ & $-6.24(1.29)^{* * *}$ & $0.06(0.02)^{* * *}$ & $-3.64(0.76)^{* * *}$ & $-0.40(0.15) *$ & $-4.03(0.76)^{* * *}$ & 9.3 \\
\hline \multicolumn{7}{|c|}{ Exposure: AL and Outcome: DSST } \\
\hline Mediator & $\begin{array}{l}\text { Exposure to } \\
\text { Mediator }\end{array}$ & $\begin{array}{l}\text { Mediator to } \\
\text { Outcome }\end{array}$ & Direct Effect & $\begin{array}{c}\text { Mediated } \\
\text { (Indirect) } \\
\text { Effect }\end{array}$ & $\begin{array}{l}\text { Total Effect } \\
\text { (Exposure to } \\
\text { Outcome) }\end{array}$ & $\begin{array}{c}\text { Proportion } \\
\text { Mediated (\%) }\end{array}$ \\
\hline DII & $0.14(0.05)^{* *}$ & $-1.69(0.30)^{* * *}$ & $-2.33(0.48)^{* * *}$ & $-0.24(0.10) *$ & $-2.57(0.48)^{* * *}$ & 9.2 \\
\hline $\begin{array}{l}\text { Vitamin D } \\
\text { (nmol/L) }\end{array}$ & $-3.80(0.84)^{* * *}$ & $0.06(0.02)^{* * *}$ & $-2.56(0.50)^{* * *}$ & $-0.24(0.09) *$ & $-2.80(0.49)^{* * *}$ & 8.1 \\
\hline
\end{tabular}

All models adjusted for sociodemographic variables (age, gender, race, education, marital status, family income/poverty ratio), health behaviors (minutes of sedentarism and smoking habit), body mass index, missing teeth and systemic status (number of chronic medical conditions, hypertension, diabetes, HDL cholesterol, LDL cholesterol, total cholesterol, triglycerides). ${ }^{*} p<0.05 ;{ }^{* *} p<0.01 ;{ }^{* * *} p<0.001$. Abbreviations: AL, attachment loss; PD, pocket depth; DII, dietary inflammatory index; DSST, Digit Symbol Substitution Test.

\subsubsection{Vitamin D Deficit}

Similarly, linear regression analyses showed that reduced levels of vitamin D also mediated the association between periodontitis and diminished cognitive function.

A negative association was found between mean AL and animal fluency test, mean PD and AL with DSST (Tables 4 and 5). Further, both mean PD and AL were negatively associated with circulating vitamin D levels. In addition, a weak association was observed between vitamin D and CERAD-DRT, animal fluency test and DSST (Tables 3-5). The mediation effect of vitamin D levels in the total association between periodontitis and cognitive function was estimated to range between $8.1 \%$ and $73.2 \%(p<0.05)$.

\section{Discussion}

In the present study, community-dwelling US older adults diagnosed with periodontitis exhibited worse cognitive function than periodontally healthy individuals. In the adjusted mediation analysis, a more inflammatory dietary load and lower vitamin D levels 
showed to significantly mediate the association of periodontitis with poor performance in all cognitive tests.

The inflammatory dietary burden was quantified through the DII, a comprehensive and literature-based tool $[37,48]$ previously linked with the variation of inflammatory surrogates [49-51] and implicated in systemic diseases [52,53]. In all, DII remarkably mediated the association of periodontitis and cognitive decline, which may be seen as novel. The role of an overly inflammatory diet in dementia has been consistently proposed [21,54,55], as it may accelerate its progression through the trigger of neuroinflammation pathways. As a result, an individual with an inflammatory imbalance due to diet or inflammatory conditions (i.e., periodontitis) may precipitate biological mechanisms that may worsen cognitive decline. Thus, resolving both factors through proper diet and periodontal treatment might have the potential to mitigate this neuroinflammatory processes, though at this stage this is merely speculative.

Additionally, the association of low circulating levels of vitamin $\mathrm{D}$ with periodontitis has been well studied $[29,56]$. The same was found for dementia, where individuals with vitamin D deficiency have a higher risk of cognitive impairment and dementia $[57,58]$. In fact, vitamin $\mathrm{D}$ regulates calcium balance, $\mathrm{A} \beta$ deposition and has antioxidant and anti-inflammatory properties in Alzheimer's disease [55,59].

Overall, this study presents methodological strengths and limitations that deserve consideration. The periodontal diagnosis was based on a full-mouth examination of six sites per tooth, which is considered the gold-standard approach, with low bias risk $[45,60,61]$. This contrasts with a previous study where the partial-mouth inspection was carried out [8]. Furthermore, cognitive function was assessed through the application of four tests, which enlightens the perception of the individual cognitive status. Further, the presence of diabetes and hypertension was confirmed through recognized clinical standards, despite the presence of the remaining pathologies that were based on self-reports. On the other hand, the cross-sectional design of the NHANES limits any extrapolation of causality or temporal association. The NHANES 2011-2012 and 2013-2014 lack gingival bleeding data, precluding a more exhaustive analysis on periodontal inflammation and the computation of the periodontal inflamed surface area that was previously linked in the association of periodontitis and cognitive functioning [8]. In addition, the DII score was the result of 26 out of 45 possible food parameters and, therefore, this could contribute to the underestimation of these results, yet this approach has been previously employed [62,63]. Also, the food questionnaire concerns a self-report from the past $24 \mathrm{~h}$ span, and this may be seen as a limitation [51]. Long-term prospective studies and well-designed interventional trials are warranted to enlighten the association between periodontitis and cognitive decline and how the inflammatory dietary burden and vitamin $\mathrm{D}$ levels can exert such a mediation effect.

\section{Conclusions}

Periodontitis was associated with significantly worse cognitive performance, and periodontitis patients reported a proinflammatory prone diet. Furthermore, serum vitamin D levels were decreased in periodontitis patients. Ultimately, the link between periodontitis and an impaired cognitive function seems to be mediated both by a proinflammatory dietary load and vitamin D deficits. Future studies should further explore the periodontitiscognitive decline link, as well as the mechanisms through which the inflammatory dietary burden and low vitamin D levels are involved in this association.

Supplementary Materials: The following are available online at https:/ / www.mdpi.com/2072-664 3/13/3/924/s1, Table S1: STROBE checklist.

Author Contributions: Conceptualization, J.B. (João Botelho), Y.L., J.V. and V.M.; methodology, J.B. (João Botelho), Y.L., J.V. and V.M.; software, J.B. (João Botelho), Y.L., J.V. and V.M.; validation, J.B. (João Botelho), Y.L., J.V. and V.M.; formal analysis, J.B. (João Botelho), Y.L. and J.V.; investigation, J.B. (João Botelho), Y.L., J.V. and V.M.; writing—original draft preparation, J.B. (João Botelho), Y.L., J.V., V.M., P.L., J.M.A., J.M.P.-P., J.B. (Juan Blanco), T.S. and J.J.M.; writing-review and editing, J.B. 
(Juan Blanco), Y.L., J.V., V.M., P.L., J.M.A., J.M.P.-P., J.B. (Juan Blanco), T.S. and J.J.M.; visualization, J.B. (João Botelho), Y.L., J.V. and V.M.; project administration, J.J.M. All authors have read and agreed to the published version of the manuscript.

Funding: This research received no external funding.

Institutional Review Board Statement: Ethical review and approval were waived for this study due to prior ethical clearance from regulatory institutions.

Informed Consent Statement: Informed consent was obtained from all subjects involved in the study.

Data Availability Statement: Data are available at the NHANES website.

Acknowledgments: Yago Leira has a research contract with Fundación Instituto de Investigación Sanitaria de Santiago de Compostela (fIDIS) and holds a Senior Clinical Research Fellowship supported by the UCL Biomedical Research Centre who receives funding from the NIHR (NIHR-INF-0387). Tomás Sobrino is a recipient of a research contract from Miguel Servet Program of Institute of Health Carlos III (CPII17/00027). The present analysis was partially funded by the Spanish Ministry of Economy (RTI2018-102165-B-I00 and RTC2019-007373-1), Interreg Atlantic Area (EAPA_791/2018) and European Commission under the PANA project (Call H2020-NMP-2015 two-stage, Grant 686009).

Conflicts of Interest: The authors declare no conflict of interest.

\section{References}

1. World Health Organization. Dementia. 2020. Available online: https://www.who.int/news-room/fact-sheets/detail/dementia (accessed on 19 October 2020).

2. Alzheimer's Disease International. World Alzheimer Report 2015, The Global Impact of Dementia: An Analysis of Prevalence, Incidence, Cost and Trends; Alzheimer's Disease International: London, UK, 2015; Available online: https://www.alz.co.uk/research/ WorldAlzheimerReport2015.pdf (accessed on 29 August 2020).

3. Wu, Y.-T.; Beiser, A.S.; Breteler, M.M.B.; Fratiglioni, L.; Helmer, C.; Hendrie, H.C.; Honda, H.; Ikram, M.A.; Langa, K.M.; Lobo, A.; et al. The changing prevalence and incidence of dementia over time-Current evidence. Nat. Rev. Neurol. 2017, 13, 327-339. [CrossRef]

4. Brayne, C.; Davis, D. Making Alzheimer's and dementia research fit for populations. Lancet 2012, 380, 1441-1443. [CrossRef]

5. $\quad$ Fang, W.-L.; Jiang, M.-J.; Gu, B.-B.; Wei, Y.-M.; Fan, S.-N.; Liao, W.; Zheng, Y.-Q.; Liao, S.-W.; Xiong, Y.; Li, Y.; et al. Tooth loss as a risk factor for dementia: Systematic review and meta-analysis of 21 observational studies. BMC Psychiatry 2018, 18, 1-11. [CrossRef]

6. Takeuchi, K.; Ohara, T.; Furuta, M.; Takeshita, T.; Shibata, Y.; Hata, J.; Yoshida, D.; Yamashita, Y.; Ninomiya, T. Tooth loss and risk of dementia in the community: The Hisayama Study. J. Am. Geriatr. Soc. 2017, 65, e95-e100. [CrossRef]

7. Chen, J.; Ren, C.-J.; Wu, L.; Xia, L.-Y.; Shao, J.; Leng, W.-D.; Zeng, X.-T. Tooth loss is associated with increased risk of dementia and with a dose-response relationship. Front. Aging Neurosci. 2018, 10, 415. [CrossRef] [PubMed]

8. Li, A.; Chen, Y.; van der Sluis, L.W.M.; Schuller, A.A.; Tjakkes, G.-H. White blood cell count mediates the association between periodontal inflammation and cognitive performance measured by digit symbol substitution test among older, U.S. adults. J. Gerontol. Ser. A 2009. [CrossRef]

9. Demmer, R.T.; Norby, F.L.; Lakshminarayan, K.; Walker, K.A.; Pankow, J.S.; Folsom, A.R.; Mosley, T.; Beck, J.; Lutsey, P.L. Periodontal disease and incident dementia: The Atherosclerosis Risk in Communities Study (ARIC). Neurology. 2020, 95, 1660-1671. [CrossRef]

10. Beydoun, M.A.; Beydoun, H.A.; Hossain, S.; El-Hajj, Z.W.; Weiss, J.; Zonderman, A.B. Clinical and bacterial markers of periodontitis and their association with incident all-cause and alzheimer's disease dementia in a large national survey. $J$. Alzheimer's Dis. 2020, 75, 157-172. [CrossRef]

11. Beydoun, M.A.; Beydoun, H.A.; Weiss, J.; Hossain, S.; El-Hajj, Z.W.; Zonderman, A.B. Helicobacter pylori, periodontal pathogens, and their interactive association with incident all-cause and Alzheimer's disease dementia in a large national survey. Mol. Psychiatry 2020, 1-16. [CrossRef]

12. Kim, D.-H.; Jeong, S.-N.; Lee, J.-H. Severe periodontitis with tooth loss as a modifiable risk factor for the development of Alzheimer, vascular, and mixed dementia: National Health Insurance Service-National Health Screening Retrospective Cohort 2002-2015. J. Periodontal Implant. Sci. 2020, 50, 303-312. [CrossRef] [PubMed]

13. Tzeng, N.-S.; Chung, C.-H.; Yeh, C.-B.; Huang, R.-Y.; Yuh, D.-Y.; Huang, S.-Y.; Lu, R.-B.; Chang, H.-A.; Kao, Y.-C.; Chiang, W.-S.; et al. Are chronic periodontitis and gingivitis associated with dementia? A nationwide, retrospective, matched-cohort study in Taiwan. Neuroepidemiology 2016, 47, 82-93. [CrossRef] [PubMed]

14. Ebersole, J.L.; Dawson, D.; Emecen-Huja, P.; Nagarajan, R.; Howard, K.; Grady, M.E.; Thompson, K.; Peyyala, R.; Al-Attar, A.; Lethbridge, K.; et al. The periodontal war: Microbes and immunity. Periodontology 2000 2017, 75, 52-115. [CrossRef] [PubMed]

15. Slots, J. Periodontitis: Facts, fallacies and the future. Periodontology 2000 2017, 75, 7-23. [CrossRef] [PubMed] 
16. Hajishengallis, G. Periodontitis: From microbial immune subversion to systemic inflammation. Nat. Rev. Immunol. 2015, 15, 30-44. [CrossRef]

17. Darveau, R.P. Periodontitis: A polymicrobial disruption of host homeostasis. Nat. Rev. Microbiol. 2010, 8, 481-490. [CrossRef]

18. Botelho, J.; Machado, V.; Proença, L.; Bellini, D.H.; Chambrone, L.; Alcoforado, G.; Mendes, J.J. The impact of nonsurgical periodontal treatment on oral health-related quality of life: A systematic review and meta-analysis. Clin. Oral Investig. 2020, 24, 585-596. [CrossRef]

19. Tonsekar, P.P.; Jiang, S.S.; Yue, G. Periodontal disease, tooth loss and dementia: Is there a link? A systematic review. Gerodontology 2017, 34, 151-163. [CrossRef]

20. Maldonado, A.; Laugisch, O.; Bürgin, W.; Sculean, A.; Eick, S. Clinical periodontal variables in patients with and without de-mentia-A systematic review and meta-analysis. Clin Oral Investig. 2018, 22, 2463-2474. [CrossRef]

21. Leigh, S.-J.; Morris, M.J. Diet, inflammation and the gut microbiome: Mechanisms for obesity-associated cognitive impairment. Biochim. Biophys. Acta Mol. Basis Dis. 2020, 1866, 165767. [CrossRef]

22. Moretti, R.; Peinkhofer, C. B Vitamins and fatty acids: What do they share with small vessel disease-related dementia? Int. J. Mol. Sci. 2019, 20, 5797. [CrossRef]

23. Hayden, K.M.; Beavers, D.P.; Steck, S.E.; Hebert, J.R.; Tabung, F.K.; Shivappa, N.; Casanova, R.; Manson, J.E.; Padula, C.B.; Salmoirago-Blotcher, E.; et al. The association between an inflammatory diet and global cognitive function and incident dementia in older women: The Women's Health Initiative Memory Study. Alzheimer's Dement. 2017, 13, 1187-1196. [CrossRef]

24. Ozawa, M.; Shipley, M.; Kivimaki, M.; Singh-Manoux, A.; Brunner, E.J. Dietary pattern, inflammation and cognitive decline: The Whitehall II prospective cohort study. Clin. Nutr. 2017, 36, 506-512. [CrossRef] [PubMed]

25. Woelber, J.P.; Bremer, K.; Vach, K.; König, D.; Hellwig, E.; Ratka-Krüger, P.; Al-Ahmad, A.; Tennert, C. An oral health optimized diet can reduce gingival and periodontal inflammation in humans-A randomized controlled pilot study. BMC Oral Health 2016, 17, 1-8. [CrossRef]

26. Zhao, C.; Tsapanou, A.; Manly, J.; Schupf, N.; Brickman, A.M.; Gu, Y. Vitamin D intake is associated with dementia risk in the Washington Heights-Inwood Columbia Aging Project (WHICAP). Alzheimer's Dement. 2020, 16. [CrossRef]

27. Soysal, P.; Dokuzlar, O.; Erken, N.; Günay, F.S.D.; Isik, A.T. The relationship between dementia subtypes and nutritional parameters in older adults. J. Am. Med. Dir. Assoc. 2020, 21, 1430-1435. [CrossRef] [PubMed]

28. Sultan, S.; Taimuri, U.; Basnan, S.A.; Ai-Orabi, W.K.; Awadallah, A.; Almowald, F.; Hazazi, A. Low vitamin d and its association with cognitive impairment and dementia. J. Aging Res. 2020, 2020, 6097820. [CrossRef]

29. Machado, V.; Lobo, S.; Proença, L.; Mendes, J.J.; Botelho, J. Vitamin D and periodontitis: A systematic review and meta-analysis. Nutrients 2020, 12, 2177. [CrossRef]

30. Von Elm, E.; Altman, D.G.; Egger, M.; Pocock, S.J.; Gøtzsche, P.C.; Vandenbroucke, J.P. The strengthening the reporting of obser-vational studies in epidemiology (STROBE) statement: Guidelines for reporting observational studies. PLoS Med. 2007, 4, 1623-1627. [CrossRef] [PubMed]

31. Brody, D.J.; Kramarow, E.A.; Taylor, C.A.; McGuire, L.C. Cognitive performance in adults aged 60 and Over: National Health and Nutrition Examination Survey, 2011-2014. Natl. Health. Stat. Rep. 2019, 126, 1-23.

32. Moms, J.C.; Heyman, A.; Mohs, R.C.; Hughes, J.P.; Van Belle, G.; Fillenbaum, G.; Mellits, E.D.; Clark, C. The consortium to establish a registry for alzheimer's disease (CERAD). Part I. Clinical and neuropsychological assessment of Alzheimer's disease. Neurology 1989, 39, 1159. [CrossRef]

33. Strauss, E.; Sherman, E.; Spreen, O. A Compendium of Neuropsychological Tests: Administration, Norms and Commentary; Oxford University Press: New York, NY, USA, 2006.

34. Salthouse, T.A. What do adult age differences in the digit symbol substitution test reflect? J. Gerontol. 1992, 47, P121-P128. [CrossRef]

35. Dye, B.A.; Thornton-Evans, G. A Brief history of national surveillance efforts for periodontal disease in the United States. J. Periodontol. 2007, 78, 1373-1379. [CrossRef] [PubMed]

36. Eke, P.I.; Page, R.C.; Wei, L.; Thornton-Evans, G.; Genco, R.J. Update of the case definitions for population-based surveillance of periodontitis. J. Periodontol. 2012, 83, 1449-1454. [CrossRef] [PubMed]

37. Shivappa, N.; Steck, S.E.; Hurley, T.G.; Hussey, J.R.; Hébert, J.R. Designing and developing a literature-derived, population-based dietary inflammatory index. Public Health Nutr. 2014, 17, 1689-1696. [CrossRef]

38. Muñoz Aguilera, E.; Leira, Y.; Miró Catalina, Q.; Orlandi, M.; Czesnikiewicz-Guzik, M.; Guzik, T.J.; Hingorani, A.D.; Nart, J.; D'Aiuto, F. Is systemic inflammation a missing link between periodontitis and hypertension? Results from two large populationsbased surveys. J. Intern. Med. 2020. [CrossRef]

39. Beck, J.D.; Moss, K.L.; Morelli, T.; Offenbacher, S. Periodontal profile class is associated with prevalent diabetes, coronary heart disease, stroke, and systemic markers of C-reactive protein and interleukin-6. J. Periodontol. 2018, 89, 157-165. [CrossRef] [PubMed]

40. Liu, Y.; Yu, Y.; Nickel, J.C.; Iwasaki, L.R.; Duan, P.; Simmer-Beck, M.; Brown, L. Gender differences in the association of periodontitis and type 2 diabetes. Int. Dent. J. 2018, 68, 433-440. [CrossRef]

41. Williams, B.; Mancia, G.; Spiering, W.; Agabiti Rosei, E.; Azizi, M.; Burnier, M.; Clement, D.L.; Coca, A.; de Simmone, G.; Dominiczak, A.; et al. 2018 ESC/ESH Guidelines for the management of arterial hypertension. Eur. Heart J. 2018, 39, 3021-3104. [CrossRef]

42. Kopelman, P.G. Obesity as a medical problem. Nature 2000, 404, 635-643. [CrossRef] 
43. Center for Disease Control and Prevention. MEC Laboratory Procedures Manual; Center for Disease Control and Prevention: Atlanta, GA, USA, 2016. Available online: https://www.cdc.gov/nchs/data/nhanes/nhanes_15_16/2016_MEC_Laboratory_ Procedures_Manual.pdf (accessed on 30 August 2020).

44. Center for Disease Control and Prevention. NHANES Laboratory Procedures Manual; Center for Disease Control and Prevention: Atlanta, GA, USA, 2009. Available online: https://www.cdc.gov/nchs/data/nhanes/nhanes_09_10/lab.pdf (accessed on 30 August 2020).

45. Botelho, J.; Machado, V.; Proença, L.; Mendes, J.J. The 2018 periodontitis case definition improves accuracy performance of full-mouth partial diagnostic protocols. Sci. Rep. 2020, 10, 1-7. [CrossRef]

46. Botelho, J.; Lyra, P.; Proença, L.; Godinho, C.; Mendes, J.J.; Machado, V. Relationship between blood and standard biochemistry levels with periodontitis in parkinson's disease patients: Data from the NHANES 2011-2012. J. Pers. Med. 2020, 10, 69. [CrossRef]

47. Leira, Y.; Carballo, Á.; Orlandi, M.; Aldrey, J.M.; Pías-Peleteiro, J.M.; Moreno, F.; Vázquez-Vázquez, L.; Campos, F.; D’Aiuto, F.; Castillo, J.; et al. Periodontitis and systemic markers of neurodegeneration: A case-control study. J. Clin. Periodontol. 2020, 47, 561-571. [CrossRef]

48. Cavicchia, P.P.; Steck, S.E.; Hurley, T.G.; Hussey, J.R.; Ma, Y.; Ockene, I.S.; Hébert, J.R. A new dietary inflammatory index predicts interval changes in serum high-sensitivity c-reactive protein. J. Nutr. 2009, 139, 2365-2372. [CrossRef]

49. Carson, S.J.; Burns, J. Impact of smoking on tooth loss in adults. Evid. Based Dent. 2016, 17, 73-74. [CrossRef]

50. Niegawa, T.; Takitani, K.; Takaya, R.; Ishiro, M.; Kuroyanagi, Y.; Okasora, K.; Minami, Y.; Matsuda, T.; Tamai, H. Evaluation of uric acid levels, thyroid function, and anthropometric parameters in Japanese children with Down syndrome. J. Clin. Biochem. Nutr. 2018, 62, 179-186. [CrossRef] [PubMed]

51. Shivappa, N.; Hebert, J.R.; Marcos, A.; Diaz, L.E.; Gomez, S.; Nova, E.; Michels, N.; Arouca, A.; González-Gil, E.; Frederic, G.; et al. Association between dietary inflammatory index and inflammatory markers in the HELENA study. Mol. Nutr. Food Res. 2017, 61, 1-23. [CrossRef]

52. Ramallal, R.; Toledo, E.; Martínez, J.A.; Shivappa, N.; Hébert, J.R.; Martínez-González, M.A.; Ruiz-Canela, M. Inflammatory potential of diet, weight gain, and incidence of overweight/obesity: The SUN cohort. Obesity 2017, 25, 997-1005. [CrossRef] [PubMed]

53. Neufcourt, L.; Assmann, K.E.; Fezeu, L.; Touvier, M.; Graffouillère, L.; Shivappa, N.; Hebert, J.R.; Wirth, M.D.; Hercberg, S.; Galan, P.; et al. Prospective association between the dietary inflammatory index and metabolic syndrome: Findings from the SU.VI.MAX study. Nutr. Metab. Cardiovasc. Dis. 2015, 25, 988-996. [CrossRef]

54. Kheirouri, S.; Alizadeh, M. Dietary inflammatory potential and the risk of neurodegenerative diseases in adults. Epidemiol. Rev. 2019, 41, 109-120. [CrossRef] [PubMed]

55. Vasefi, M.; Hudson, M.; Ghaboolian-Zare, E. Diet Associated with Inflammation and Alzheimer's Disease. J. Alzheimer's Dis. Rep. 2019, 3, 299-309. [CrossRef]

56. Botelho, J.; Machado, V.; Proença, L.; Delgado, A.S.; Mendes, J.J. Vitamin D deficiency and oral Health: A comprehensive review. Nutrients 2020, 12, 1471. [CrossRef]

57. Etgen, T.; Chonchol, M.; Förstl, H.; Sander, D. Chronic kidney disease and cognitive impairment: A Systematic review and meta-analysis. Am. J. Nephrol. 2012, 35, 474-482. [CrossRef] [PubMed]

58. Balion, C.; Griffith, L.E.; Strifler, L.; Henderson, M.; Patterson, C.; Heckman, G.; Llewellyn, D.J.; Raina, P. Vitamin D, cognition, and dementia: A systematic review and meta-analysis. Neurology 2012, 79, 1397-1405. [CrossRef] [PubMed]

59. Landel, V.; Annweiler, C.; Millet, P.; Morello, M.; Féron, F. Vitamin D, cognition and alzheimer's Disease: The therapeutic benefit is in the d-tails. J. Alzheimer's Dis. 2016, 53, 419-444. [CrossRef]

60. Tran, D.T.; Gay, I.; Du, X.L.; Fu, Y.; Bebermeyer, R.D.; Neumann, A.S.; Streckfus, C.; Chan, W.; Walji, M.F. Assessing periodontitis in populations: A systematic review of the validity of partial-mouth examination protocols. J. Clin. Periodontol. 2013, 40, 1064-1071. [CrossRef]

61. Eke, P.; Thornton-Evans, G.; Wei, L.; Borgnakke, W.; Dye, B. Accuracy of NHANES periodontal examination protocols. J. Dent. Res. 2010, 89, 1208-1213. [CrossRef]

62. Deng, F.E.; Shivappa, N.; Tang, Y.; Mann, J.R.; Hebert, J.R. Association between diet-related inflammation, all-cause, all-cancer, and cardiovascular disease mortality, with special focus on prediabetics: Findings from NHANES III. Eur. J. Nutr. 2017, 56, 1085-1093. [CrossRef] [PubMed]

63. Kotsakis, G.A.; Chrepa, V.; Shivappa, N.; Wirth, M.; Hébert, J.; Koyanagi, A.; Tyrovolas, S. Diet-borne systemic inflammation is associated with prevalent tooth loss. Clin. Nutr. 2018, 37, 1306-1312. [CrossRef] [PubMed] 